\title{
ПОДХОД К СТРУКТУРНО-ФУНКЦИОНАЛЬНОМУ МОДЕЛИРОВАНИЮ МОРФОЛОГИЧЕСКОЙ СТРУКТУРЫ ПОВЕРХНОСТИ МАТЕРИАЛОВ НА ОСНОВЕ ФТОРИРОВАННЫХ ПОЛИМЕРОВ
}

\author{
Г.О. Рытиков, Ф. А. Доронин, А. Г. Евдокимов, М. А. Савельев, В. Г. Назаров \\ ГЕОРГИЙ ОЛЕГОВИЧ РЫТИКОВ - к.ф.-м.н., дочент Московского политехнического университета. \\ E-mail: gr-yandex@yandex.ru. \\ ФЕДОР АЛЕКСАНДРОВИЧ ДОРОНИН - стариий преподаватель Московского политехнического \\ университета.E-mail: f.a.doronin@mospolytech.ru.
}

АНДРЕЙ ГРИГОРЬЕВИЧ ЕВДОКИМОВ - старший преподаватель Московского политехнического университета.E-mail: evdokag@gmail.com.

МИХАИЛ АЛЕКСАНДРОВИЧ САВЕЛЬЕВ - к.т.н., стариий научный сотрудник Межведомственного иентра аналитических исследований в области физики, химии и биологии при Президиуме Российской академии наук.E-mail:vidogo@yandex.ru.

ВИКТОР ГЕННАДЬЕВИЧ НАЗАРОВ - д.т.н., профессор Московского политехнического университета. E-mail:110505n@gmail.com.

107023, г. Москва, Большая Семеновская ул., 38, Московский политехнический университет.

117997, г. Москва, ул. Профсоюзная, д. 65, с.6, ФГБУН Межведомственный центр аналитических исследований в области физики, химии и биологии при Президиуме Российской академии наук.

В работе осуществлено структурно-динамическое моделирование полимодальной морфологической структуры поверхностей полимеров на примере модифичрованного полиэтилентерефталата. В частности, продемонстрированы скорректированные значения амплитуд гармоник Фурье-разложения, позволяющие моделировать морфологию поверхности фторированных полимерных пленок с более высокой точностью.

Ключевые слова: фторирование, математическое моделирование, полимеры, морфология

\section{APPROACH TO STRUCTURAL AND FUNCTIONAL MODELING OF THE MORPHOLOGICAL STRUCTURE OF THE SURFACE OF MATERIALS BASED ON FLUORINATED POLYMERS}

\author{
G. O. Rytikov ${ }^{1}$ F.A. Doronin ${ }^{1}$, A. G. Evdokimov ${ }^{1}$, M.A. Savelev², V. G. Nazarov ${ }^{1}$ \\ 138, Bolshaya Semyonovskaya str., Moscow, Moscow Polytechnic University. \\ 265/6, Profsouznaya st., Moscow, Interdepartmental Center of Analytical Research in Physics, Chemistry \\ and Biology at the Presidium of the Russian Academy of Sciences.
}


In this paper structural and dynamic modeling of the polymodal morphological structure of polymer surfaces is performed on the example of modified polyethylene terephthalate. In particular, the corrected values of the amplitudes of the Fourier expansion harmonics are demonstrated, which allow us to model the surface morphology of fluorinated polymer films with higher accuracy.

Keywords: fluorination, mathematical modeling, polymers, morphology

Известно [1], что высокомолекулярные соединения широко используют в различных отраслях промышленности в качестве конструкционных и функциональных материалов, эксплуатация которых осуществляется, в том числе, в экстремальных условиях [2], что, свою очередь, предъявляет особые требования к их эксплуатационным свойствам (хим-, биостойкость и тп. [3, 4]). В связи с этим возрастает потребность в материалах с типичным для полимеров (но ещё не достигнутым) набором свойств [5]. Однако, вариативность физико-химических и функциональных характеристик полимеров настолько велика, что, зачастую возникает необходимость в синтезе материала, обладающего взаимоисключающими свойствами. Концептуальным решением данной проблемы (по аналогии с тем, как это было сделано при создании сплавов металлов) является модификация существующих полимеров для придания им необходимых функциональных свойств [6]. Так, объёмная модификация позволяет конструировать материал наподобие твёрдого раствора: свойства объёмно модифицированных полимеров преимущественно однородны в любом элементе их объёмов и могут всюду отличаться от свойств ингредиентов. В свою очередь, свойства поверхностно модифицированных полимеров существенно отличаются на поверхности и в объеме образца [7]. При поверхностной модификации фактически происходит формирование тонкого слоя одного полимера на поверхности другого, причём чёткая граница раздела между слоями отсутствует. Существуют такие способы [8] поверхностной модификации, как прививка сополимеров, жидко- или газофазная модификация [9], плазмохимическая обработка [10] и др. Таким образом, «объёмные» свойства полимер фактически наследует от полимерной матрицы, а поверхностные - от формируемого при модификации квазидвумерного компонента. Это исключительно удобно для решения ряда задач микрофлюидики, гибкой электроники и планарной фотоники [11].

Одним из основных инструментов визуализации морфологии поверхностей в т.ч. модифицированных полимеров в настоящее время являются изображения, формируемые средствами скани- рующей электронной микроскопии [12]. В отличие от высокоразрешающей оптической и атомносиловой микроскопии (ориентированной на визуализацию микрорельефа) СЭМ обеспечивает наиболее полную характеристику поверхности, учитывающей как микрорельеф, так и химическое строение полимеров. Именно химический потенциал поверхности полимеров определяет существенную часть эксплуатационных и иных свойств изделий на их основе. В некотором смысле оказывается не очень важным, каковы именно особенности микрорельефа или характер распределения химических элементов по поверхности полимера, если известно на основе эксперимента как именно исследуемая поверхность будет взаимодействовать с окружающей средой. При этом СЭМ-изображения часто характеризуются грубо статистически путём применения встроенного программного обеспечения, поставляемого вместе с измерительным оборудованием.

Количественный анализ СЭМ-изображений производится достаточно редко, обычно всё ограничивается анализом визуально-качественным, т.к. для большинства известных практических применений достаточно убедиться в наличии некоторых структурных маркеров на субмикроразмерном масштабе. Основные методы количественного анализа СЭМ-изображений [13] разделяют на три группы: визуально-эмпирические (с помощью стандартных инструментов обработки изображения осуществляются измерения геометрических размеров тех или иных одиночных наблюдаемых исследователем объектов), структурно-аналитические (неоднородности микрорельефа представляются в виде суперпозиции элементов одного из известных наборов базисных функций) и структурно-стохастические (на основании сравнения статистических распределений яркостей пикселей выявляются структурные домены, энергетические характеристики которых эквивалентны, несмотря на возможные геометрические отличия их друг от друга). Наиболее удобным с точки зрения простоты и наглядности моделирования, интерпретации и структурнофункционального анализа нам представляется разложение цифровых образов, анализируемых изображения в двумерный ряд Фурье. 
При решении ряда задач коллоидной химии перспективными оказались [14] микроканалы и микрокапилляры, формируемые на поверхностях оксифторированного полиэтилентерефталата (ПЭТ), поскольку модификация газовой смесью фтора и кислорода позволяет формировать на его поверхности супергидрофильную структуру, обеспечивающую, в свою очередь, самостоятельное и целенаправленное движение капель водосодержащих жидкостей по микроканалам без применения микронасосов или создания постоянного градиента давления. Таким образом, возникает необходимость в количественной аналитической характеризации и моделирования морфологии поверхности ПЭТ вследствие его оксифторирования.

\section{Объекты исследования}

В качестве объектов исследования использовали пленки ПЭТ, оксифторирование которых проводили в течение $5,30,60$ и 180 мин газовой смесью $7,5 \% \mathrm{~F}_{2} / 10 \% \mathrm{O}_{2} / 82,5 \% \mathrm{He}$, а также осуществляли исследование поверхностных свойств и химического состава ПЭТ до и после модификации, что изложено в работе [15]. СЭМ-изображения ПЭТ пленок формировали на автоэмиссионном сканирующем электронном микроскопе JSM 7500 (JEOL, Япония) (рис. 1).

\section{Теория}

Для аналитической характеризации морфологии поверхности ПЭТ удобно использовать разложение в двумерный тригонометрический ряд Фурье:

$f(x, y)=f_{0}+\sum_{m=1}^{N} \sum_{n=1}^{N}\left\{\begin{array}{l}a_{m n} \cos \left(m k_{x} x\right) \cos \left(n k_{y} y\right)+ \\ +b_{m n} \cos \left(m k_{x} x\right) \sin \left(n k_{y} y\right)+ \\ +c_{m n} \sin \left(m k_{x} x\right) \cos \left(n k_{y} y\right)+ \\ +d_{m n} \sin \left(m k_{x} x\right) \sin \left(n k_{y} y\right)\end{array}\right\},(1)$

в котором $f_{0}$ - среднее значение функции $f(x, y) ; m$, $n$ - индексы порядка бигармоник; $a_{m n}, b_{m n}, c_{m n}, d_{m n}$ частные амплитуды и $m k_{x} x, m k_{y} y$ - фазы бигармоник.

Каждая поверхность

$A_{m n}(x, y)=a_{m n} \cos \left(m k_{x} x\right) \cdot \cos \left(n k_{y} y\right)+$

$+b_{m n} \cos \left(m k_{x} x\right) \cdot \sin \left(n k_{y} y\right)+c_{m n} \sin \left(m k_{x} x\right) \cdot \cos \left(n k_{y} y\right)+$

$+d_{m n}^{m n} \sin \left(m k_{x} x\right) \cdot \sin \left(n k_{y} y\right)$,

интерпретируется как пространственная решетка с периодами $\lambda_{x}=2 \pi / \mathrm{k}_{x}$ и $\lambda_{y}=2 \pi / \mathrm{k}_{y}$ соответственно. Совокупность $\left\{A_{m n}\right\}_{\mathrm{N}^{2}}$ таких решеток обеспечивает формирование модели морфологического спектра, с высокой точностью описывающего структуру поверхности ПЭТ пленки.
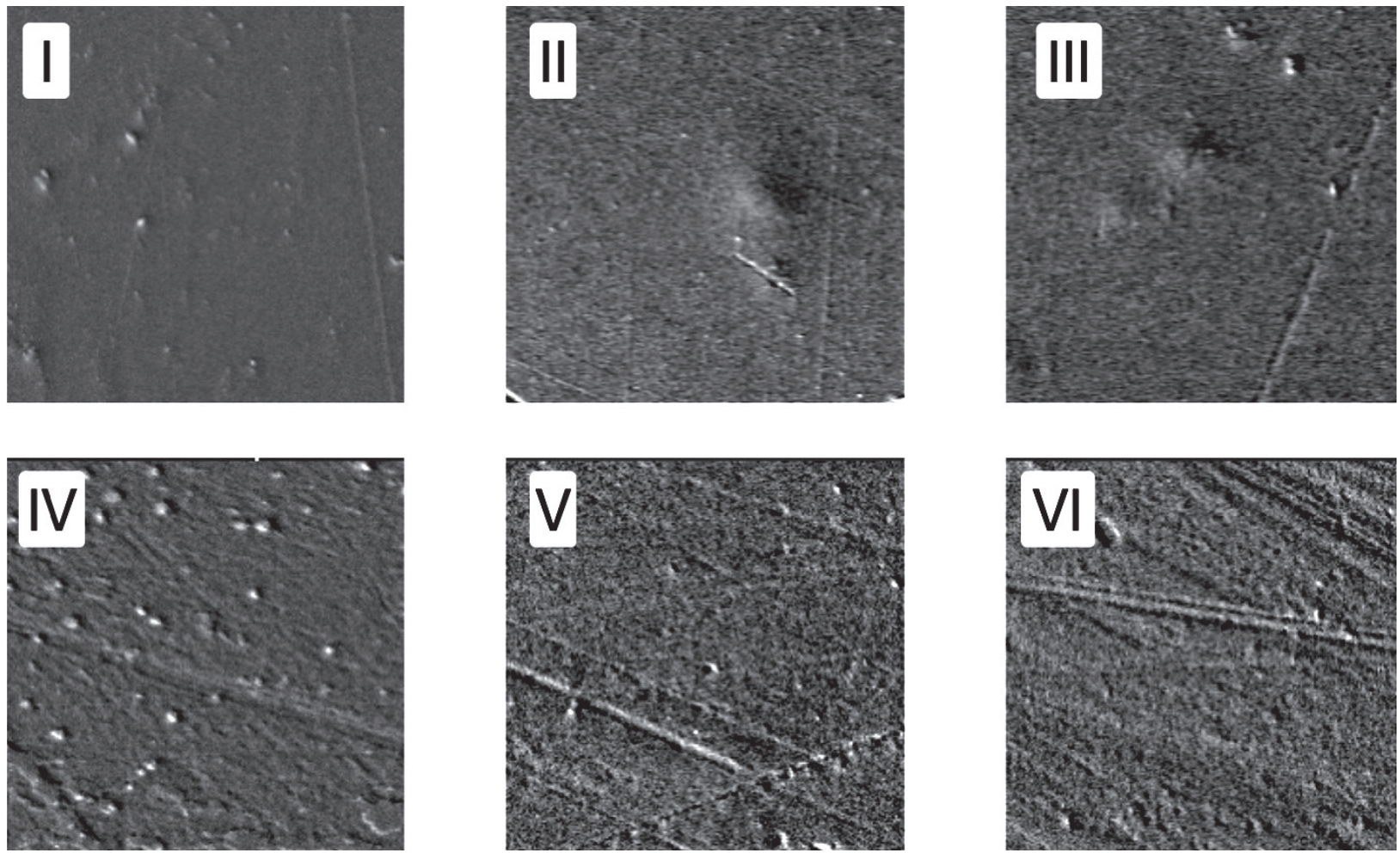

Рис. 1. СЭМ-изображения ПЭТ пленок:

исходной (I) и оксифторированных в течение 2 (II), 5 (III), 30 (IV), 60 (V) и 180 (VI) мин 
При разложении заданной на некотором интервале периодической функции в одномерный ряд Фурье обычно выделяют амплитудную и фазовую составляющие спектра. В этом случае амплитуда каждой гармоники полагается равной корню квадратному из суммы квадратов её компонент. В первом приближении в качестве оценочного значения амплитуд бигармоник двумерного Фурье-спектра также можно ограничиться выражением $A_{m n}=\sqrt{\left|a_{m n}\right|^{2}+\left|b_{m n}\right|^{2}+\left|c_{m n}\right|^{2}+\left|d_{m n}\right|^{2}}$. Однако для установления точного значения соответствующих амплитуд необходимо осуществить корректировку этого выражения.

Рассмотрим функцию:

$z(x, y)=a \cdot \cos x \cdot \cos y+b \cdot \cos x \cdot \sin y+c \cdot \sin x \cdot \cos y+$ $d \cdot \sin x \cdot \sin y$

Для того чтобы найти экстремальные значения $\left\{z\left(x_{0}, y_{0}\right)\right\}_{\min ; \max }$ этой функции, необходимо решить систему уравнений и найти значение $z(x, y)$ в соответствующих точках тт. $\left\{M\left(x_{0}, y_{0}\right)\right\}_{\min ; \max }$ :

$$
\left\{\begin{array}{l}
\left.(\partial z / \partial x)\right|_{y=\text { const }}=0 \\
\left.(\partial z / \partial y)\right|_{x=\text { const }}=0
\end{array} \Rightarrow\left\{M\left(x_{0}, y_{0}\right)\right\} \Rightarrow\left\{z\left(x_{0}, y_{0}\right)\right\}\right.
$$

При непосредственном дифференцировании функции $z(x, y)$ в форме (3) соответствующий алгоритм (4) не позволяет найти аналитическое решение системы уравнений явно. Использование стандартных тригонометрических преобразований вида $\cos \alpha \cdot \cos \beta=\frac{1}{2}(\cos (\alpha+\beta)+\cos (\alpha-\beta))$ позволяет перейти к представлению:

$$
\begin{aligned}
& z=\frac{1}{2} \cdot((a-d) \cdot \cos (x+y)+(a+d) \cdot \cos (x-y)+ \\
& +(b+c) \cdot \sin (x+y)+(c-b) \cdot \sin (x-y))
\end{aligned}
$$

и привести систему уравнений (2) к виду:

$$
\left\{\begin{array}{l}
x+y=\operatorname{arctg}((b+c) /(a-d))+\pi \cdot n \\
x-y=\operatorname{arctg}((c-b) /(a+d))+\pi \cdot n,
\end{array} n \in Z\right.
$$

Таким образом, координаты особых точек $\left\{M\left(x_{0}, y_{0}\right)\right\}$ принимают вид

$$
\begin{aligned}
& x_{0}=\frac{1}{2} \cdot(\operatorname{arctg}((b+c) /(a-d))+\operatorname{arctg}((c-b) /(a+d))) \\
& y_{0}=\frac{1}{2} \cdot(\operatorname{arctg}((b+c) /(a-d))-\operatorname{arctg}((c-b) /(a+d)))
\end{aligned}
$$

и значения функции $z(x, y)$ в этих точках определяются выражением:

$$
z\left(x_{0}, y_{0}\right)=\frac{1}{2} \cdot A \cdot(\sqrt{1+\phi}+\sqrt{1-\phi}),
$$

в котором введены обозначения

$$
\begin{aligned}
& A=\sqrt{a^{2}+b^{2}+c^{2}+d^{2}}, \\
& \Delta=a \cdot d-b \cdot c, \\
& \phi=\frac{2 \cdot(a \cdot d-b \cdot c)}{a^{2}+b^{2}+c^{2}+d^{2}}=\frac{2 \cdot \Delta}{A}
\end{aligned}
$$

Здесь $z\left(x_{0}, y_{0}\right)$ является амплитудной, а параметр $\varphi$ интерпретируется как фазовая характеристика наблюдаемой на конкретном изображении частной реализации пространственной решётки $A_{11}$.

Простой анализ функции (5) на экстремум по переменной $\varphi$ :

$$
\frac{d z}{d \phi}=\frac{1}{2} \cdot A \cdot\left(\frac{1}{2 \sqrt{1+\phi}}-\frac{1}{2 \sqrt{1-\phi}}\right)=0 \quad \& \quad \phi \in[-1 ; 1](9)
$$

показывает, что:

$$
\begin{aligned}
& z_{\text {max }}(\phi=0)=\sqrt{a^{2}+b^{2}+c^{2}+d^{2}} \\
& z_{\text {min }}(\phi= \pm 1)=\frac{1}{\sqrt{2}} \cdot \sqrt{a^{2}+b^{2}+c^{2}+d^{2}}
\end{aligned}
$$

T.e., в зависимости от конкретной фазовой реализации, наблюдаемое значение амплитуды гармоники может принимать значения в диапазоне от $A_{m n} / \sqrt{2}$ до $A_{m n}$. Таким образом, для адекватного описания морфологии поверхности с помощью разложения в двумерный ряд Фурье необходимо осуществлять корректировку полученных значений амплитуд гармоник, вычисляя для каждой гармоники поправочный коэффициент:

$$
\kappa_{m n}=\frac{2}{\sqrt{1+\phi_{m n}}+\sqrt{1-\phi_{m n}}}
$$

В [13] нами был описан комплексный метод характеризации поверхностей, а также приведены морфологические спектры без соответствующих поправок. В данной статье демонстрируются скорректированные значения амплитуд гармоник Фурье-разложения, позволяющие моделировать морфологию поверхности с более высокой точностью. 

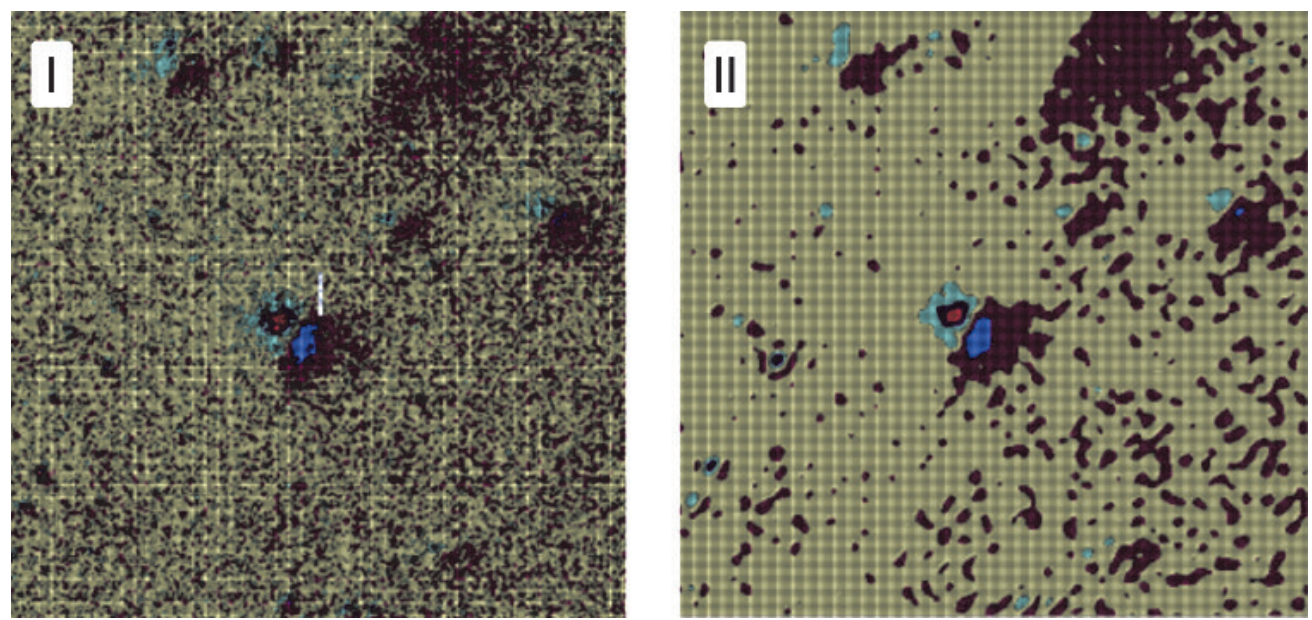

Рис. 2. Цифровой образ СЭМ изображений ПЭТ пленок (I) и результат его восстановления по морфологическому спектру с учетом фазовых поправок (II)

\section{Результаты и их обсуждение}

Цифровые образы исходной и восстановленной с учетом фазовых поправок поверхностей плёнки ПЭТ представлены на рис. 2.

Видно, что структура морфологических спектров сохранилась, однако соотношения между отдельными амплитудами изменились. При этом качество восстановления поверхности выше при учёте фазовых поправок.

Количественной мерой качества восстановления поверхности традиционно является коэффициент детерминации:

$$
R^{2}=1-\frac{\sum_{i=1}^{N_{x}} \sum_{j=1}^{N_{y}}\left(f_{i j}-f_{i j, c a l c}\right)^{2}}{\sum_{i=1}^{N_{x}} \sum_{j=1}^{N_{y}}\left(f_{i j}-\bar{f}\right)^{2}}
$$

где $\bar{f}=\frac{1}{N_{x} N_{y}} \cdot \sum_{i=1}^{N_{x}} \sum_{j=1}^{N_{y}} f_{i j}-$ средняя яркость пикселей анализируемого изображения; $f_{i j}, f_{i j, c a l c}-$ значения яркостей пикселей исходного и восстановленного изображений, $N_{x}, N_{y}$ - выраженные в пикселях горизонтальный и вертикальный размеры анализируемого изображения.
Значения коэффициентов детерминации, полученных при аппроксимации модельными разложениями в двумерный ряд Фурье цифровых образов СЭМ-изображений ПЭТ пленок, полученных в результате оксифторирования в течение $0,2,5$, 30, 60 и 180 мин, представлены в таблице.

Таким образом, учёт фазовых поправок повышает качество аппроксимации экспериментальных данных теоретическими моделями приблизительно на $20 \%$. Соответствующие морфологические спектры, характеризующие поверхности оксифторированных ПЭТ пленок, представлены на рис. 3.

Видно, что наибольшие значения амплитуд морфологического спектра наблюдаются при пятиминутной (рис. 3.III) продолжительности оксифторирования, а наибольшее количество статистически значимых амплитуд наблюдается при тридцатиминутном оксифторировании (рис. 3.IV).

Таким образом, наибольшая эффективность оксифторирования наблюдается в диапазоне продолжительностей модификации 5-30 мин, а дальнейшее оксифторирование к существенному изменению морфологии модифицируемой поверхности не приводит.

Исследование выполнено при финансовой поддержке РФФИ в рамках гранта № 20-03-00191.

Качество аппроксимации экспериментальных данных

\begin{tabular}{ccccccc}
\hline $\mathrm{t}$, мин & 0 & 2 & 5 & 30 & 60 & 180 \\
\hline $\mathrm{R}^{2}$ & 0,22 & 0,57 & 0,51 & 0,40 & 0,31 & 0,31 \\
\hline $\mathrm{R}_{\text {corr }}^{2}$ & 0,27 & 0,68 & 0,62 & 0,48 & 0,38 & 0,37 \\
\hline
\end{tabular}



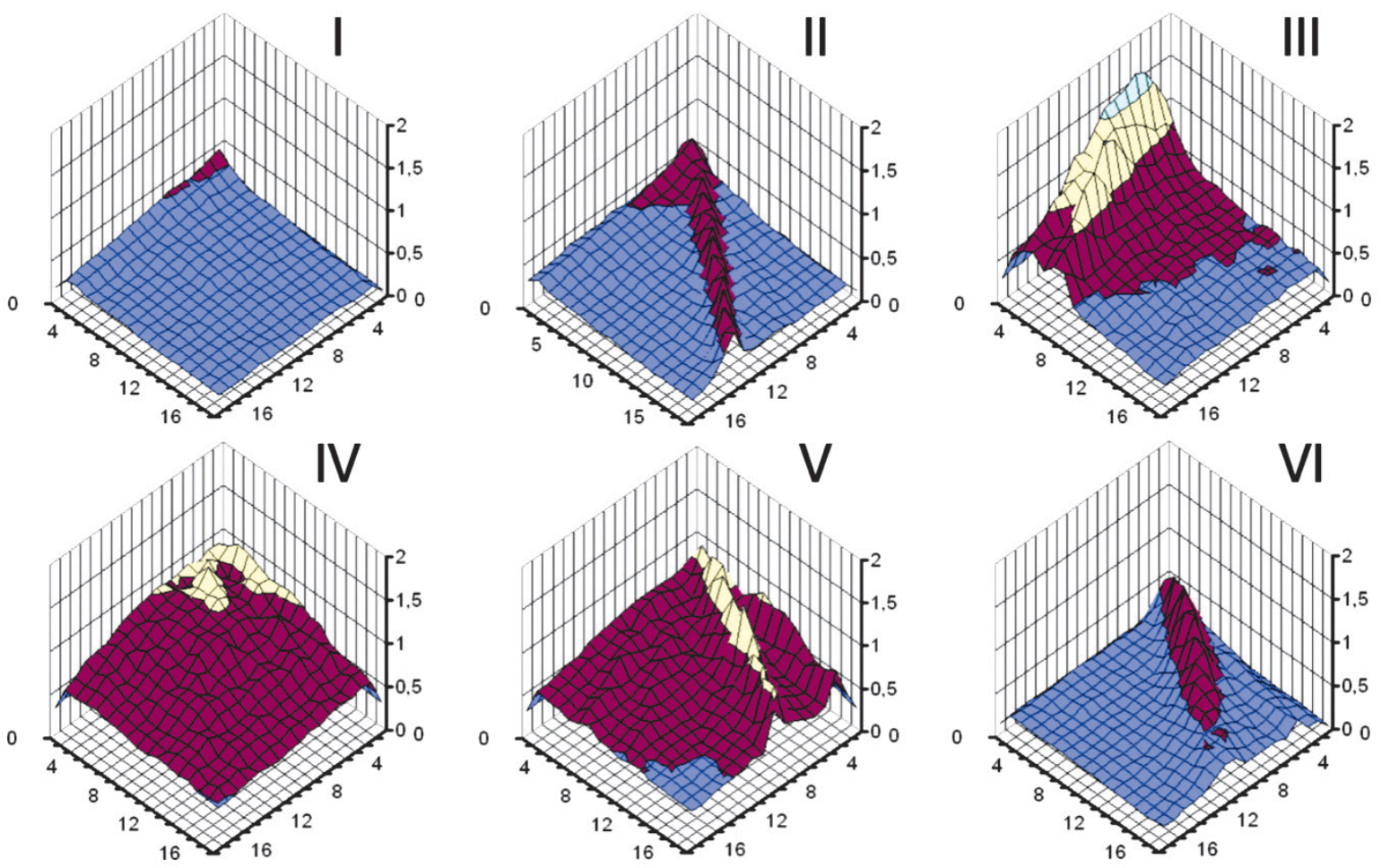

Рис. 3. Нескорректированные морфологические спектры ПЭТ пленок: исходной (I) и оксифторированных в течение 2 (II), 5 (III), 30 (IV), 60 (V) и 180 (VI) мин

\section{Лuтература}

1. Da'Silva R.M.P., Mano J.F., Reis R.L. Trends in Biotechnology. 2007. V. 25. P. 577-583.

2. Каблов Е.Н., Стариев О.В. Авиационные материалы и технологии. 2015. №4 (37). С. 38-52.

3. Huang J., Matyjaszewski K., Koepsel R.R., Murata H., Russell A.J. Biomacromolecules. 2007. T. 8. №5. P. 1396-1399.

4. Lichter J.A., Van Vlietpa K.J., Rubner M.F. Macromolecules. 2009. T. 42. №22. P. 8573-8586.

5. Gao J., Xu X., Fan C., Wang X., Dai Y., Liu X.. Materials Letters. 2014. V. 121. P. 219-222.

6. Үи S., Ни H., Ma J., Yin J. Tribology International. 2008. V. 41. P. 1205-1211.

7. Nazarov V.G., Nagornova I.V., Stolyarov V.P., Doronin F.A., Evdokimov A.G., Brevnov P.N., Zabolotnov A.S., Novokshonova L.A. Russ. J. Phys. Chem. B. 2018. V. 12. P. 1066-1075.
8. Kharitonov A.P. Prog. Org. Coat. 2008. V. 61. P. 192204.

9. Nazarov V.G. J. Appl. Polym. Sci. 2005. V. 95. P. 897902.

10. Yoshida S., Hagiwara K., Hasebe T., Hotta A. Surface and Coatings Technology. 2013. V. 233. P. 99-107.

11. Becker H., Locascio L.E. Polymer microfluidic devices. Talanta. 2002. V. 56. Iss. 2. P. 267-287.

12. Pease R.F.W. Advances in Imaging and Electron Physics. Elsevier. 2008. V. 150. P. 53-86.

13. Копачев Е.С., Ноздрачев С.А., Петрушин В.Н., Рудяк Ю.В., Рытиков Г.О., Назаров В.Г. Физическая мезомеханика. 2015. Т. 18. № 6. С. 98-110.

14. Doronin F.A., Nagornova I.V., Rytikov G.O., Varepo L.G., Nazarov V.G. J. Phys.: Conf. Ser. 2020. V. 1546. P. 012007.

15. Nazarov V.G., Doronin F.A., Evdokimov A.G., Stolyarov V.P. Colloid J. 2019. V. 81. P. 146-157. 